\title{
Graded Cube of Opposition with Intermediate Quantifiers in Fuzzy Natural Logic
}

\author{
Petra Murinová( $\left.{ }^{\otimes}\right)$ and Vilém Novák \\ Institute for Research and Applications of Fuzzy Modeling, \\ University of Ostrava, 30. dubna 22, 70103 Ostrava 1, Czech Republic \\ \{petra.murinova, vilem.novak\}@osu.cz \\ http://irafm.osu.cz/
}

\begin{abstract}
In our previous papers, we formally analyzed the generalized Aristotle's square of opposition using tools of higher-order fuzzy logic. Namely, we introduced general definitions of selected intermediate quantifiers, constructed a generalized square of opposition consisting of them and syntactically analyzed the emerged properties. The main objective of this paper is to extend the graded Peterson's square of opposition into the graded cube of opposition with intermediate quantifiers.
\end{abstract}

Keywords: Intermediate quantifiers $\cdot$ Fuzzy natural logic $\cdot$ Evaluative linguistic expressions $\cdot$ Generalized peterson square $\cdot$ Graded cube of opposition

\section{Introduction}

This paper continues the work on intermediate quantifiers. Fuzzy natural logic (FNL) is a formal mathematical theory that consists of three theories: (1) a formal theory of evaluative linguistic expressions explained in detail in [1], (2) a formal theory of fuzzy IF-THEN rules and approximate reasoning presented in $[2,3]$, and (3) a formal theory of intermediate and generalized fuzzy quantifiers presented in [4-7]. This paper is a contribution to the latter.

Intermediate quantifiers are special linguistic expressions, for example, almost all, a few, many, a large part of, etc. which were introduced and deeply studied by Thompson in [8] and later by Peterson in his book in [9]. Peterson introduced a square of opposition as a generalization of the Aristotle's one [10-13]. It consists of five basic intermediate quantifiers.

Formalization of Peterson's square was introduced by Murinová and Novák in $[14,15]$. The main objective of this paper is to extend this approach to a graded 5-cube of opposition and prove its forming properties.

The work was supported from ERDF/ESF by the project "Centre for the development of Artificial Intelligence Methods for the Automotive Industry of the region" No. CZ.02.1.01/0.0/0.0/17-049/0008414 and partially also by the MŠMT project NPU II project LQ1602 "IT4Innovations excellence in science".

(C) Springer Nature Switzerland AG 2020

M.-J. Lesot et al. (Eds.): IPMU 2020, CCIS 1239, pp. 145-158, 2020.

https://doi.org/10.1007/978-3-030-50153-2_11 
Note that the idea to extend the square of opposition to a cube was already studied by Dubois in [16-18]. The authors introduced a graded Aristotle's square of opposition extended to a cube that associates the traditional square of opposition with a dual one. Ciucci, Dubois, and Prade [19,20] then introduced an application of the graded cube within the possibility theory.

\section{Preliminaries}

In this section, we will remind the main concepts and properties of the fuzzy type theory (higher-order fuzzy logic) and the theory of evaluative linguistic expressions. The reader can find details in several papers $[1,14,21]$.

\subsection{Fuzzy Type Theory}

The formal theory of intermediate quantifiers is developed within Łukasiewicz fuzzy type theory (Ł-FTT). The algebra of truth values is a linearly ordered $\mathrm{MV}_{\boldsymbol{\Delta}}$-algebra extended by the delta operation (see $\left.[22,23]\right)$. A special case is the standard Łukasiewicz $\mathrm{MV}_{\Delta}$-algebra.

$$
\mathcal{L}=\langle[0,1], \vee, \wedge, \otimes, \rightarrow, 0,1, \Delta\rangle
$$

where

$$
\begin{aligned}
\wedge & =\text { minimum, } & \vee & =\text { maximum, } \\
a \otimes b & =0 \vee(a+b-1), & a \rightarrow b & =1 \wedge(1-a+b), \\
\neg a & =a \rightarrow 0=1-a, & \Delta(a) & = \begin{cases}1 & \text { if } a=1, \\
0 & \text { otherwise. }\end{cases}
\end{aligned}
$$

The basic syntactical objects of E-FTT are classical (cf. [24]), namely the concepts of type and formula. The atomic types are $\epsilon$ (elements) and $o$ (truth values). General types are denoted by Greek letters $\alpha, \beta, \ldots$ We will omit the type whenever it is clear from the context. A set of all types is denoted by Types. The (meta-)symbol ":=" used below means "is defined by".

The language consists of variables $x_{\alpha}, \ldots$, special constants $c_{\alpha}, \ldots(\alpha \in$ Types), symbol $\lambda$, and parentheses. The connectives (which are special constants) are fuzzy equality/equivalence $\equiv$, conjunction $\wedge$, implication $\Rightarrow$, negation $\neg$, strong conjunction \&, strong disjunction $\boldsymbol{\nabla}$, disjunction $\mathrm{V}$, and delta $\boldsymbol{\Delta}$.

Formulas are formed of variables, constants (each of specific type), and the symbol $\lambda$. Each formula $A$ is assigned a type and we write it as $A_{\alpha} \cdot{ }^{1}$ A set of formulas of type $\alpha$ is denoted by Form $_{\alpha}$. The set of all formulas is Form = $\bigcup_{\alpha \in \text { Types }}$ Form $_{\alpha}$.

\footnotetext{
${ }^{1}$ Each formula has a unique type assigned to it. Hence, if $\alpha, \beta$ are different types then $A_{\alpha}$ and $A_{\beta}$ are different formulas. To increase clarity of explanation, however, we will usually denote different formulas by different letters.
} 
A model is $\mathcal{M}=\left\{\left(M_{\alpha}, \stackrel{\circ}{=}_{\alpha}\right) \mid \alpha \in\right.$ Types $\}$ where $\stackrel{\circ}{=}_{\alpha}$ is a fuzzy equality on a set $M_{\alpha}$. If $\mathcal{M}$ is a model then $\mathcal{M}\left(A_{o}\right) \in M_{o}$ is a truth value, $\mathcal{M}\left(A_{\epsilon}\right) \in M_{\epsilon}$ is some element and $\mathcal{M}\left(A_{\beta \alpha}\right): M_{\alpha} \rightarrow M_{\beta}$ is a function. For example, $\mathcal{M}\left(A_{o \alpha}\right)$ : $M_{\alpha} \rightarrow M_{o}$ is a fuzzy set and $\mathcal{M}\left(A_{(o \alpha) \alpha}\right): M_{\alpha} \times M_{\alpha} \rightarrow M_{o}$ a fuzzy relation. A formula $A_{o}$ is true in $T, T \models A_{o}$, if it is true in the degree 1 in all models of $T$.

The fuzzy type theory is complete, i.e., a theory $T$ is consistent iff it has a (Henkin) model. We sometimes apply its equivalent version: $T \vdash A_{o}$ iff $T \models A_{o}$.

The following special formulas play a role in our theory below:

$$
\begin{array}{cc}
\Upsilon_{o o} \equiv \lambda z_{o} \cdot \neg \Delta\left(\neg z_{o}\right), & \text { (nonzero truth value) } \\
\hat{\Upsilon}_{o o} \equiv \lambda z_{o} \cdot \neg \Delta\left(z_{o} \vee \neg z_{o}\right) . & \text { (general truth value) }
\end{array}
$$

Thus, $\mathcal{M}\left(\Upsilon\left(A_{o}\right)\right)=1$ iff $\mathcal{M}\left(A_{o}\right)>0$, and $\mathcal{M}\left(\hat{\Upsilon}\left(A_{o}\right)\right)=1$ iff $\mathcal{M}\left(A_{o}\right) \in(0,1)$ holds in any model $\mathcal{M}$.

\subsection{Theory of Evaluative Linguistic Expressions}

Evaluative linguistic expressions are expressions of a natural language such as small, medium, big, very short, more or less deep, quite roughly strong, extremely high, etc. Their theory is the basic constituent of the fuzzy natural logic.

The semantics of evaluative linguistic expressions is formulated in a special formal theory $T^{\mathrm{Ev}}$ of E-FTT that was introduced in [1] and less formally explained in [25] where also formulas for direct computation are provided.

The evaluative expressions are construed by special formulas $S m \in$ Form $_{\text {oo(oo) }}$ (small), Me $\in$ Form $_{\text {oo(oo) }}$ (medium), Bi $\in$ Form $_{\text {oo(oo) }}$ (big), and $Z e \in$ Form $_{\text {oo (oo) }}$ (zero) that can be extended by several selected linguistic hedges. Recall that a hedge, i.e., usually (but not necessarily) an adverb such as "very, significantly, about, roughly", etc. is in general construed by a formula $\boldsymbol{\nu} \in$ Form $_{\text {oo }}$ with specific properties. To classify that a given formula is a hedge, we introduced a formula Hedge $\in$ Form $_{o(o o)}$. Then $T^{\mathrm{Ev}} \vdash$ Hedge $\boldsymbol{\nu}$ means that $\boldsymbol{\nu}$ is a hedge. We refer the reader to [1] for the technical details. We assume that the following is provable: $T^{\mathrm{Ev}} \vdash$ Hedge $\boldsymbol{\nu}$ for all $\boldsymbol{\nu} \in\{E x, S i, V e, M L, R o, Q R, V R\}$.

\subsection{Theory of Intermediate Quantifiers}

The theory of intermediate quantifiers is a special formal theory $T^{\mathrm{IQ}}[\mathcal{S}]$ of E-FTT extending $T^{\mathrm{Ev}}$. A detailed structure of $T^{\mathrm{IQ}}[\mathcal{S}]$ and precise definitions can be found in $[5,6,14]$.

As discussed in the Introduction, the semantics of the intermediate quantifiers requires the idea of a "size" of a (fuzzy) set that can be characterized by the concept of a measure. 
Definition 1. Let $R \in$ Form $_{o(o \alpha)(o \alpha)}$ be a formula ${ }^{2}$.

(i) A formula $\mu \in$ Form $_{o(o \alpha)(o \alpha)}$ defined by

$$
\mu_{o(o \alpha)(o \alpha)}:=\lambda z_{o \alpha} \lambda x_{o \alpha}\left(R z_{o \alpha}\right) x_{o \alpha}
$$

represents a measure on fuzzy sets in the universe of type $\alpha \in$ Types if it has the following properties:

(M1) $\boldsymbol{\Delta}\left(x_{o \alpha} \subseteq z_{o \alpha}\right) \& \Delta\left(y_{o \alpha} \subseteq z_{o \alpha}\right) \& \Delta\left(x_{o \alpha} \subseteq y_{o \alpha}\right) \Rightarrow\left(\left(\mu z_{o \alpha}\right) x_{o \alpha} \Rightarrow\right.$ $\left.\left(\mu z_{o \alpha}\right) y_{o \alpha}\right)$

(M2) $\Delta\left(x_{o \alpha} \subseteq z_{o \alpha}\right) \Rightarrow\left(\left(\mu z_{o \alpha}\right)\left(z_{o \alpha} \backslash x_{o \alpha}\right) \equiv \neg\left(\mu z_{o \alpha}\right) x_{o \alpha}\right)$,

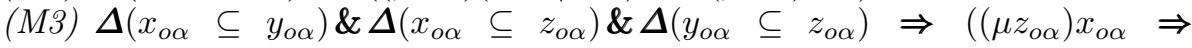
$\left.\left(\mu y_{o \alpha}\right) x_{o \alpha}\right)$.

(ii) The following formula characterizes measurable fuzzy sets of a given type $\alpha$ :

$$
\begin{aligned}
& \mathbf{M}_{o(o \alpha)}:=\lambda z_{o \alpha} \cdot \Delta \neg\left(z_{o \alpha} \equiv \emptyset_{o \alpha}\right) \& \Delta\left(\mu z_{o \alpha}\right) z_{o \alpha} \& \\
&\left(\forall x_{o \alpha}\right)\left(\forall y_{o \alpha}\right) \Delta((M 1) \&(M 3)) \&\left(\forall x_{o \alpha}\right) \Delta(M 2)
\end{aligned}
$$

where, for the simplicity of expression, we write (M1)-(M3) to stand for the axioms from (i).

Definition 2. Let $\mathcal{S} \subseteq$ Types be a selected set of types, $P=\{R \in$ Form $\left._{o(o \alpha)(o \alpha)} \mid \alpha \in \mathcal{S}\right\}$ be a set of new constants. Let $T$ be a consistent extension of the theory $T^{E v}$ in the language $J(T) \supseteq J^{E v} \cup P$. We say that the theory $T$ contains intermediate quantifiers w.r.t. the set of types $\mathcal{S}$ if for all $\alpha \in \mathcal{S}$ the following is provable:

(i)

$$
T \vdash\left(\exists z_{o \alpha}\right) \mathbf{M}_{o(o \alpha)} z_{o \alpha}
$$

(ii)

$$
T \vdash\left(\forall z_{o \alpha}\right)\left(\exists x_{o \alpha}\right)\left(\mathbf{M}_{o(o \alpha)} z_{o \alpha} \Rightarrow\left(\Delta\left(x_{o \alpha} \subseteq z_{o \alpha}\right) \& \hat{\Upsilon}\left(\left(\mu z_{o \alpha}\right) x_{o \alpha}\right)\right) .\right.
$$

Formula (5) assures the existence of fuzzy sets in each measurable fuzzy set that have non-trivial measure. Obviously, formulas (4) and (5) can be also introduced as special axioms of $T$. In the sequel, we will denote a theory that contains intermediate quantifiers due to Definition 2 by $T^{\mathrm{IQ}}$.

For the definition of intermediate quantifiers, we need to define a special operation called cut of a fuzzy set, which will be formally defined as follows: Let $y, z \in$ Form $_{o \alpha}$. The cut of $y$ by $z$ is the fuzzy set

$$
y \mid z \equiv \lambda x_{\alpha} \cdot z x \& \Delta(\Upsilon(z x) \Rightarrow(y x \equiv z x)) .
$$

The following lemma can be proved.

\footnotetext{
${ }^{2}$ This formula can be understood as a procedure providing computation of the output (a value in $L$ ) on the basis of a given input - two fuzzy sets. Formula (2) says that the measure is a function.
} 
Lemma 1 ([15]). Let $\mathcal{M}$ be a model and $p$ an assignment such that $B=$ $\mathcal{M}_{p}(y) \lesssim M_{\alpha}, Z=\mathcal{M}_{p}(z) \subsetneq M_{\alpha}$. Then for any $m \in M_{\alpha}$

$$
\mathcal{M}_{p}(y \mid z)(m)=(B \mid Z)(m)= \begin{cases}B(m), & \text { if } B(m)=Z(m), \\ 0 & \text { otherwise. }\end{cases}
$$

One can see that the operation $B \mid Z$ takes only those elements $m \in M_{\alpha}$ from the fuzzy set $B$ whose membership $B(m)$ is equal to $Z(m)$, otherwise $(B \mid Z)(m)=0$. If there is no such an element, then $B \mid Z=\emptyset$. We can thus use various fuzzy sets $Z$ to "picking up proper elements" from $B$.

The following lemma will play a significant role in proofs of properties of the graded cube of opposition.

Lemma 2. Let $\mathcal{M}$ be a model and $p$ an assignment such that $B=\mathcal{M}_{p}(y) \subsetneq M_{\alpha}$, $Z=\mathcal{M}_{p}(z) \Subset M_{\alpha}, Z^{\prime}=\mathcal{M}_{p}\left(z^{\prime}\right) \cong M_{\alpha}$. Then for any $m \in M_{\alpha}$

(a) $(B \mid Z)(m) \otimes\left(\neg B \mid Z^{\prime}\right)(m)=0$,

(b) $(B \mid Z)(m) \otimes \neg\left(B \mid Z^{\prime}\right)(m)=0$.

Proof. (a) Let $p(x)=m$ and $B(m)=0$. Then the property is trivially fulfilled.

Let $B(m)=Z(m)>0$ and $\neg B(m)=Z^{\prime}(m)>0$. Then from Lemma 1 it follows that $B(m)=(B \mid Z)(m)$ as well as $\neg B(m)=\left(\neg B \mid Z^{\prime}\right)(m)$. Because $B(m) \otimes \neg B(m)=1$ holds for any $m \in M_{\alpha}$, then $(B \mid Z)(m) \otimes\left(\neg B \mid Z^{\prime}\right)(m)=0$.

(b) Obviously as (a).

Definition 3. Let $T^{I Q}$ be a theory containing intermediate quantifiers w.r.t. a set of types $\mathcal{S}$ due to Definition 2. Let $E v \in$ Form $_{\text {oo }}$ be an intension of some evaluative expression. Finally, let $z \in$ Form $_{o \alpha}, x \in$ Form $_{\alpha}$ be variables and $A, B \in$ Form $_{o \alpha}$ be formulas where $T^{I Q} \vdash \mathbf{M}_{o(o \alpha)} B_{o \alpha}, \alpha \in \mathcal{S}$. An intermediate generalized quantifier construes the sentence " $\langle$ Quantifier $\rangle$ B's are $A$ " is one of the following formulas:

$$
\begin{aligned}
& \left(Q_{E v}^{\forall} x\right)(B, A) \equiv(\exists z)[(\forall x)((B \mid z) x \Rightarrow A x) \wedge E v((\mu B)(B \mid z))], \\
& \left(Q_{E v}^{\exists} x\right)(B, A) \equiv(\exists z)[(\exists x)((B \mid z) x \wedge A x) \wedge E v((\mu B)(B \mid z))] .
\end{aligned}
$$

The following special intermediate quantifiers can be introduced:

$$
\begin{aligned}
\text { A: All } B \text { are } A & :=\left(Q_{B i \Delta}^{\forall} x\right)(B, A) \equiv(\forall x)(B x \Rightarrow A x), \\
\text { E: No } B \text { are } A & :=\left(Q_{B i \Delta}^{\forall} x\right)(B, \neg A) \equiv(\forall x)(B x \Rightarrow \neg A x), \\
\text { P: Almost all } B \text { 's are } A & :=\left(Q_{B i E x}^{\forall} x\right)(B, A)
\end{aligned}
$$

B: Almost all $B$ 's are not $A:=\left(Q_{B i E x}^{\forall} x\right)(B, \neg A)$

T: Most $B$ 's are $A:=\left(Q_{B i}^{\forall} e^{x}\right)(B, A)$

D: Most $B$ 's are not $A:=\left(Q_{B i}^{\forall} e^{x}\right)(B, \neg A)$

K: Many $B$ 's are $A:=\left(Q_{\neg S m}^{\forall} x\right)(B, A)$ 
G: Many $B$ 's are not $A:=\left(Q_{\neg S m}^{\forall} x\right)(B, \neg A)$

I: Some $B$ are $A:=\left(Q_{B i \Delta}^{\exists} x\right)(B, A) \equiv(\exists x)(B x \wedge A x)$,

O: Some $B$ are not $A:=\left(Q_{B i \Delta}^{\exists} x\right)(B, \neg A) \equiv(\exists x)(B x \wedge \neg A x)$.

\section{Graded Cube of Opposition}

\subsection{From the Graded Square to the Graded Cube of Opposition}

The graded Aristotle's square of opposition is formed by two positive and two negative intermediate quantifiers that fulfil the generalized properties of contraries, contradictories, sub-contraries, and sub-alterns. Below, we recall the main definitions from [14].

Definition 4. Let $T$ be a consistent theory of $E-F T T, \mathcal{M} \models T$ be a model, $p \in A s g(\mathcal{M})$ be an assignment, and $P_{1}, P_{2} \in$ Form $_{o}$ be closed formulas of type o.

(i) $P_{1}$ and $P_{2}$ are contraries in the model $\mathcal{M}$ if

$$
\mathcal{M}_{p}\left(P_{1}\right) \otimes \mathcal{M}_{p}\left(P_{2}\right)=0 .
$$

They are contraries in the theory $T$ if $T \vdash \neg\left(P_{1} \& P_{2}\right)$. By completeness, this is equivalent to (8) for every model $\mathcal{M} \models T$.

(ii) $P_{1}$ and $P_{2}$ are subcontraries in the model $\mathcal{M}$ if

$$
\mathcal{M}_{p}\left(P_{1}\right) \oplus \mathcal{M}_{p}\left(P_{2}\right)=1 \text {. }
$$

They are subcontraries in the theory $T$ if $T \vdash\left(P_{1} \nabla P_{2}\right)$. By completeness, this is equivalent to (9) for every model $\mathcal{M} \models T$.

(iii) $P_{1}$ and $P_{2}$ are contradictories in the model $\mathcal{M}$ if both

$$
\mathcal{M}_{p}\left(\Delta P_{1}\right) \otimes \mathcal{M}_{p}\left(\Delta P_{2}\right)=0 \text { as well as } \mathcal{M}_{p}\left(\Delta P_{1}\right) \oplus \mathcal{M}_{p}\left(\Delta P_{2}\right)=1 \text {. }
$$

They are contradictories in the theory $T$ if both $T \vdash \neg\left(\Delta P_{1} \& \Delta P_{2}\right)$ as well as $T \vdash \Delta P_{1} \nabla \Delta P_{2}$. By completeness, this means that (10) hold for every model $\mathcal{M} \models T$.

(iv) $P_{2}$ is a subaltern of $P_{1}\left(P_{1}\right.$ is superaltern of $\left.P_{2}\right)$ in the model $\mathcal{M}$ if

$$
\mathcal{M}_{p}\left(P_{1}\right) \leq \mathcal{M}_{p}\left(P_{2}\right) .
$$

$P_{2}$ is subaltern of $P_{1}$ in the theory $T$ ( $P_{1}$ is superaltern of $P_{2}$ in the theory T) if $T \vdash P_{1} \Rightarrow P_{2}$. By completeness, this means that inequality (11) holds true in every model $\mathcal{M} \models T$.

All these definitions were introduced as a generalization of the corresponding classical ones. In our previous paper [14], we syntactically proved all the mentioned properties which form the graded Aristotle's square of opposition. 
Theorem 1 ([14]). The following is true in $T^{I Q}$ :

(a) formulas $\mathbf{A}$ and $\mathbf{O}$ are contradictories in $T^{I Q}$,

(b) formulas $\mathbf{E}$ and $\mathbf{I}$ are contradictories in $T^{I Q}$.

(c) formulas $\mathbf{A}$ and $\mathbf{E}$ are contraries with the presupposition in $T^{I Q}$.

(d) the formula $\mathbf{A}$ is subaltern of $\mathbf{I}$.

Changing $B$ and $A$ into their negation, $\neg B$ and $\neg A$ respectively, leads to another similar square of opposition aeio, provided that we also assume that the fuzzy set $\neg B$ is non-empty. It means that we assume presupposition ${ }^{3}$ (existential import) which was in detail discussed in our previous papers. The corresponding quantifier with presupposition is denoted by a star. To extend the graded Aristotle's square to a graded cube of opposition, we have to define new formulas as follows:

$$
\begin{array}{ll}
\text { *a: All } \neg B \text { are not } A & (\forall x)(\neg B x \Rightarrow \neg A x) \&(\exists x) \neg B x, \\
\text { e: All } \neg B \text { are } A & (\forall x)(\neg B x \Rightarrow A x), \\
\text { i: Some } \neg B \text { are not } A & (\exists x)(\neg B x \wedge \neg A x), \\
\text { *o: Some } \neg B \text { are } A & (\exists x)(\neg B x \wedge A x) \nabla \neg(\exists x) \neg B x .
\end{array}
$$

We can see that the new logical square of opposition (aeio) develops from the graded Aristotle's one (AEIO) by replacing formulas $B x$ and $A x$ by $\neg B x$ and $\neg A x$, respectively. It means that the "basic" properties between the intermediate quantifiers inside aeio can be proved similarly to AEIO.

Theorem 2. The following is true in $T^{I Q}$ :

(a) formulas a and $\mathbf{o}$ are contradictories in $T^{I Q}$,

(b) formulas $\mathbf{e}$ and $\mathbf{i}$ are contradictories in $T^{I Q}$.

(c) formulas a and $\mathbf{e}$ are contraries with the presupposition in $T^{I Q}$.

(d) formula a is subaltern of $\mathbf{i}$.

Proof. The properties (a)-(d) can be proved similarly as the properties of the graded Aristotle's square AEIO in [14].

\subsection{Relations Between "AEIO" and "aeio"}

Lemma 3. The following is provable:

(a) $T^{I Q} \vdash \neg(\mathbf{A} \& \mathbf{e})^{*}$,

(b) $T^{I Q} \vdash \neg(\mathbf{a} \& \mathbf{E})^{*}$.

\footnotetext{
${ }^{3}$ It is necessary that the universal quantifiers carry a presupposition of existential import for the entailments to their respective particular forms to hold.
} 
Proof. (a) By properties of E-FTT we have

$$
T^{\mathrm{IQ}} \vdash(B x \Rightarrow A x) \Rightarrow(\neg A x \Rightarrow \neg B x)
$$

as well as

$$
T^{\mathrm{IQ}} \vdash(\neg B x \Rightarrow A x) \Rightarrow(\neg A x \Rightarrow B x) .
$$

By $T^{\mathrm{IQ}} \vdash(\neg A x \&(\neg A x \Rightarrow B x)) \Rightarrow B x$ and $T^{\mathrm{IQ}} \vdash(\neg A x \&(\neg A x \Rightarrow$ $\neg B x)) \Rightarrow \neg B x$ we obtain the following provable formula

$$
T^{\mathrm{IQ}} \vdash(\neg A x \&(\neg A x \Rightarrow B x)) \&(\neg A x \&(\neg A x \Rightarrow \neg B x)) \Rightarrow \perp
$$

which, using the properties of E-FTT, is equivalent with

$$
T^{\mathrm{IQ}} \vdash((\neg A x \Rightarrow B x) \&(\neg A x \Rightarrow \neg B x)) \Rightarrow(\neg A x)^{2} \Rightarrow \perp
$$

From (16), (17) and (18) by transitivity and using MP, we conclude that

$$
T^{\mathrm{IQ}} \vdash((B x \Rightarrow A x) \&(\neg B x \Rightarrow A x)) \Rightarrow\left((\neg A x)^{2} \Rightarrow \perp\right) .
$$

Finally, by the quantifier properties, we have

$$
\left.T^{\mathrm{IQ}} \vdash((\forall x)(B x \Rightarrow A x) \&)(\forall x)(\neg B x \Rightarrow A x)\right) \Rightarrow\left((\exists x)(\neg A x)^{2} \Rightarrow \perp\right)
$$

which uses the definition of the negation equivalent with

$$
\left.T^{\mathrm{IQ}} \vdash \neg((\forall x)(B x \Rightarrow A x) \&)(\forall x)(\neg B x \Rightarrow A x)\right) \&(\exists x)(\neg A x)^{2}
$$

(b) It can be proved analogously.

Theorem 3. The following holds true:

(a) Formulas a and $\mathbf{E}$ are contraries in $T^{I Q}$.

(b) Formulas $\mathbf{A}$ and $\mathbf{e}$ are contraries in $T^{I Q}$.

Proof. It follows from Lemma 3.

Lemma 4. Let the following be true:

(a) $T^{I Q} \vdash(\mathbf{i} \nabla \mathbf{O})^{*}$,

(b) $T^{I Q} \vdash(\mathbf{I} \nabla \mathbf{o})^{*}$.

Proof. (a) From Lemma 3(a), we have the following provable formula

$$
\left.T^{\mathrm{IQ}} \vdash \neg((\forall x)(B x \Rightarrow A x) \&)(\forall x)(\neg B x \Rightarrow A x)\right) \&(\exists x)(\neg A x)^{2} .
$$

Then by properties of the negation, we have

$$
\left.T^{\mathrm{IQ}} \vdash(\exists x) \neg(B x \Rightarrow A x) \nabla(\exists x) \neg(\neg B x \Rightarrow A x)\right) \nabla \neg(\exists x)(\neg A x)^{2}
$$

which is equivalent with

$$
\left.T^{\mathrm{IQ}} \vdash(\exists x)(B x \boldsymbol{\&} \neg A x) \nabla(\exists x)(\neg B x \& \neg A x)\right) \nabla \neg(\exists x)(\neg A x)^{2} .
$$

Finally by properties of $\&$ with $\wedge$, we conclude that

$$
\left.T^{\mathrm{IQ}} \vdash(\exists x)(B x \wedge \neg A x) \nabla(\exists x)(\neg B x \wedge \neg A x)\right) \nabla \neg(\exists x)(\neg A x)^{2} .
$$

(b) Analogously as (a). 
Theorem 4. The following is true:

(a) Formulas $\mathbf{i}$ and $\mathbf{O}$ are subcontraries in $T^{I Q}$.

(b) Formulas $\mathbf{I}$ and $\mathbf{o}$ are subcontraries in $T^{I Q}$.

Proof. It follows from Lemma 4.

Lemma 5. The following is provable:

(a) $T^{I Q} \vdash \mathbf{A}^{*} \Rightarrow \mathbf{i} \quad T^{I Q} \vdash \mathbf{a} \Rightarrow \mathbf{I}$,

(b) $T^{I Q} \vdash \mathbf{E} \Rightarrow \mathbf{o}^{*} \quad T^{I Q} \vdash \mathbf{e} \Rightarrow \mathbf{O}$.

Proof. (a) By the properties of $\mathrm{E}-\mathrm{FTT}$, we have

$$
T^{\mathrm{IQ}} \vdash(\forall x)(B x \Rightarrow A x) \Rightarrow(\forall x)(\neg A x \Rightarrow \neg B x)
$$

as well as

$$
T^{\mathrm{IQ}} \vdash(\forall x)(\neg A x \Rightarrow \neg B x) \Rightarrow((\exists x) \neg A x \Rightarrow(\exists x)(\neg A x \wedge \neg B x)) .
$$

Finally, by (21) and (22), we obtain

$$
T^{\mathrm{IQ}} \vdash(\forall x)(B x \Rightarrow A x) \&(\exists x) \neg A x \Rightarrow(\exists x)(\neg A x \wedge \neg B x) .
$$

(b) is proved analogously.

Theorem 5. The following is true:

(a) Formula $\mathbf{A}$ is subaltern of $\mathbf{i}$, and $\mathbf{I}$ is superaltern of $\mathbf{a}$.

(b) Formula $\mathbf{E}$ is subaltern of $\mathbf{o}$, and $\mathbf{e}$ is superaltern of $\mathbf{O}$.

Proof. It follows from Lemma 5.

Example 1 (Interpretation of Cube of opposition in $T^{\mathrm{IQ}}$ ).

(a) Construction of AEIO as follows:

Let us consider a model $\mathcal{M} \models T^{\mathrm{IQ}}$ such that $T^{\mathrm{IQ}} \vdash(\exists x) B x$. Let $\mathcal{M}(\mathbf{A})=a>0$ (e.g., $a=0.2)$. Since $[\mathbf{A}, \mathbf{E}]$ are contraries, we have $\mathcal{M}(\mathbf{E})=e \leq 1-a$. Because the formulas $[\mathbf{A}, \mathbf{O}]$ are contradictories, it follows from the definition of contradictories that $\mathcal{M}(\boldsymbol{\Delta} \mathbf{A})=0$ and so $\mathcal{M}(\Delta \mathbf{O})=1$ because $\mathcal{M}(\Delta \mathbf{A}) \otimes \mathcal{M}(\Delta \mathbf{O})=0$ and $\mathcal{M}(\boldsymbol{\Delta} \mathbf{A}) \oplus \mathcal{M}(\boldsymbol{\Delta} \mathbf{O})=1$. Consequently, $\mathbf{O}$ is subaltern of $\mathbf{E}$. The $\mathbf{I}$ is subaltern of $\mathbf{A}$ and thus $\mathcal{M}(\mathbf{I})=i \geq 0.2$. However, $\mathbf{I}$ is contradictory with $\mathbf{E}$ and so $\mathcal{M}(\mathbf{I})=i=1$. Finally, $\mathbf{I}$ is sub-contrary with $\mathbf{O}$ because $\mathcal{M}(\mathbf{O} \nabla \mathbf{I})=1$ and $\mathbf{I}$ is subaltern of $\mathbf{A}$.

(b) Construction of aeio as follows:

Let us consider the same model $\mathcal{M} \models T^{\mathrm{IQ}}$ such that $T^{\mathrm{IQ}} \vdash(\exists x) \neg A x$. Let $\mathcal{M}(\mathbf{a})=a^{\prime}>0$ (e.g., $\left.a^{\prime}=0.8\right), \mathcal{M}(\mathbf{e})=e^{\prime}, \mathcal{M}(\mathbf{i})=i^{\prime}$ and $\mathcal{M}(\mathbf{o})=o^{\prime}$. Since $[\mathbf{A}, \mathbf{e}]$, as well as $[\mathbf{E}, \mathbf{i}]$ are contraries, we have $\mathcal{M}(\mathbf{e})=e^{\prime} \leq 0.2$ and $\mathcal{M}(\mathbf{a})=a^{\prime} \leq 0.8$. Similarly, formulas $[\mathbf{i}, \mathbf{O}]$ and $[\mathbf{I}, \mathbf{o}]$ are sub-contraries in $T^{\mathrm{IQ}}$ then $\mathcal{M}(i)=i^{\prime}=1$ as well as $\mathcal{M}(i)=i^{\prime}=1$. Both of these results correspond with contradictories of the pairs $[\mathbf{a}, \mathbf{o}]$ and $[\mathbf{e}, \mathbf{i}]$. Finally, $\mathbf{A}$ is subaltern of $\mathbf{i}$ as well as $\mathbf{E}$ is subaltern of $\mathbf{o}$.

These results are summarized in the following scheme. Recall that the straight lines mark contradictories, the dashed lines contraries, and the dotted lines subcontraries. The arrows indicate the relation superaltern-subaltern (Fig. 1). 


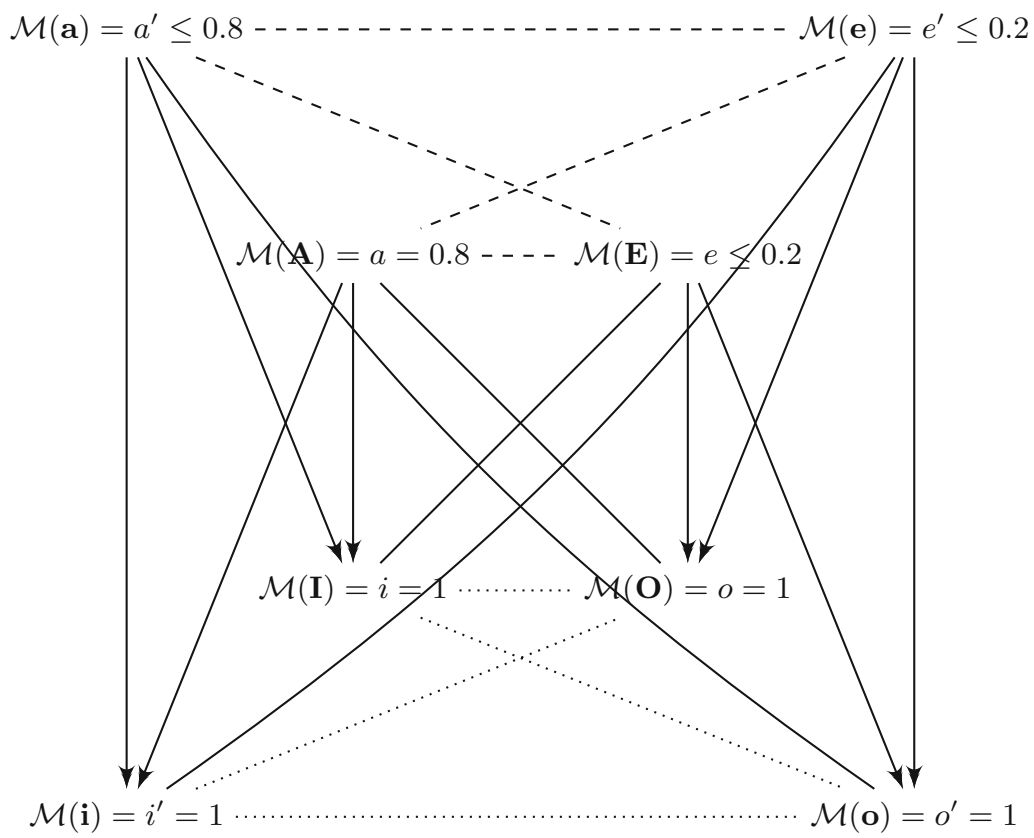

Fig. 1. The example of graded Aristotle's cube of opposition

\section{Graded Cube with Intermediate Quantifiers}

We continue with an extension of graded the 5-square of opposition AEPBTDKGIO, which was introduced as a generalization of Peterson's square, to the graded 5-cube of opposition aepbtdkgio with intermediate quantifiers. Below we introduce new forms of intermediate quantifiers as follows:

$$
\begin{aligned}
& \left(Q_{E v}^{\forall} x\right)(\neg B, \neg A) \equiv(\exists z)[(\forall x)((\neg B \mid z) x \Rightarrow \neg A x) \wedge E v((\mu(\neg B))(\neg B \mid z))], \\
& \left(Q_{E v}^{\exists} x\right)(\neg B, \neg A) \equiv(\exists z)[(\exists x)((\neg B \mid z) x \wedge \neg A x) \wedge E v((\mu(\neg B))(\neg B \mid z))] .
\end{aligned}
$$

Either of the quantifiers (23) or (24) construes the sentence

"〈Quantifier $\rangle$ not $B$ 's are not $A "$.

\subsection{Contraries}

Lemma 6. Let $B \in$ Form $_{o \alpha}$ be a formula and $z, z^{\prime} \in$ Form $_{o \alpha}$ be variables. Then the following is provable:

$$
\begin{aligned}
& T^{I Q} \vdash \neg\left[(\exists z)\left(\exists z^{\prime}\right)[(\forall x)((B \mid z) x \Rightarrow A x) \wedge E v((\mu B)(B \mid z)) \&\right. \\
& \left.\quad(\forall x)\left(\left(\neg B \mid z^{\prime}\right) x \Rightarrow A x\right) \wedge E v\left((\mu(\neg B))\left(\left(\neg B \mid z^{\prime}\right)\right)\right) \&(\exists x)(\neg(A x))^{2}\right] .
\end{aligned}
$$


Proof. The proof of (25) is based on the following provable formulas:

$$
T^{\mathrm{IQ}} \vdash((B \mid z) x \Rightarrow A x) \Rightarrow(\neg A x \Rightarrow \neg(B \mid z) x)
$$

and

$$
T^{\mathrm{IQ}} \vdash\left(\left(\neg B \mid z^{\prime}\right) x \Rightarrow A x\right) \Rightarrow\left(\neg A x \Rightarrow \neg\left(\neg B \mid z^{\prime}\right) x\right) .
$$

Using quantifier properties and by Lemma 2 we obtain from these formulas that

$$
T^{\mathrm{IQ}} \vdash(\forall x)((B \mid z) x \Rightarrow A x) \&(\forall x)\left(\left(\neg B \mid z^{\prime}\right) x \Rightarrow A x\right) \Rightarrow\left((\exists x)(\neg A x)^{2} \Rightarrow \perp\right) .
$$

By the adjunction, the properties of $\wedge$, the quantifier properties and the definition of negation, we obtain (25) using the rules of L-FTT.

Theorem 6. The pairs of quantifiers

(i) $\left[\left(Q_{B i E x}^{\forall} x\right)(B, A),\left(Q_{B i E x}^{\forall} x\right)(\neg B, A)\right]$, (i.e., $\left.[\mathbf{P}, \mathbf{b}]\right)$,

(ii) $\left[\left(Q_{B i \text { Ve }}^{\forall} x\right)(B, A),\left(Q_{B i V e}^{\forall} x\right)(\neg B, A)\right]$, (i.e., $\left.[\mathbf{T}, \mathbf{d}]\right)$,

(iii) $\left[\left(Q_{\neg(S m \overline{\boldsymbol{\nu}})}^{\forall} x\right)(B, A),\left(Q_{\neg(S m \overline{\boldsymbol{\nu}})}^{\forall} x\right)(B, \neg A)\right]$, (i.e., $\left.[\mathbf{K}, \mathbf{g}]\right)$,

are contraries in $T^{I Q}$.

Proof. It follows from Lemma 6 when replacing $E v$ by concrete evaluative linguistic expressions.

Lemma 7. Let $B \in$ Form $_{o \alpha}$ be a formula and $z, z^{\prime} \in$ Form $_{o \alpha}$ be variables. Then the following is provable:

$$
\begin{aligned}
& T^{I Q} \vdash \neg\left[(\exists z)\left(\exists z^{\prime}\right)[(\forall x)((\neg B \mid z) x \Rightarrow \neg A x) \wedge E v((\mu(\neg B))((\neg B \mid z))) \&\right. \\
&(\forall x)\left(\left(B \mid z^{\prime}\right) x \Rightarrow \neg A x\right)\left.\wedge E v\left((\mu B)\left(B \mid z^{\prime}\right)\right) \&(\exists x)(\neg(A x))^{2}\right] .
\end{aligned}
$$

Proof. Similarly to Lemma 6 , the proof of (27) is based on the following provable formula:

$$
T^{\mathrm{IQ}} \vdash(\forall x)((\neg B \mid z) x \Rightarrow \neg A x) \&(\forall x)\left(\left(B \mid z^{\prime}\right) x \Rightarrow \neg A x\right) \Rightarrow\left((\exists x)(A x)^{2} \Rightarrow \perp\right) .
$$

By the adjunction, the properties of $\wedge$, the quantifier properties and the definition of negation, we obtain (28) using the rules of E-FTT.

Theorem 7. The pairs of quantifiers

(i) $\left[\left(Q_{B i E x}^{\forall} x\right)(\neg B, \neg A),\left(Q_{B i E x}^{\forall} x\right)(B, \neg A)\right]$, (i.e., [p, B] $)$,

(ii) $\left[\left(Q_{B i \text { Ve }}^{\forall} x\right)(\neg B, \neg A),\left(Q_{B i \text { Ve }}^{\forall} x\right)(B, \neg A)\right]$, (i.e., $\left.[\mathbf{t}, \mathbf{D}]\right)$,

(iii) $\left[\left(Q_{\neg(S m \overline{\boldsymbol{\nu}})}^{\forall} x\right)(\neg B, \neg A),\left(Q_{\neg(S m \overline{\boldsymbol{\nu}})}^{\forall} x\right)(B, \neg A)\right]$, (i.e., $\left.[\mathbf{k}, \mathbf{G}]\right)$,

are contraries in $T^{I Q}$.

Proof. It follows from Lemma 7 when replacing $E v$ by concrete evaluative linguistic expressions. 


\subsection{Sub-alterns}

Lemma 8. The following is provable in E-FTT:

(a) $T^{I Q} \vdash \mathbf{a} \Rightarrow \mathbf{p} \quad T^{I Q} \vdash \mathbf{e} \Rightarrow \mathbf{b}$,

(b) $T^{I Q} \vdash \mathbf{p} \Rightarrow \mathbf{t} \quad T^{I Q} \vdash \mathbf{b} \Rightarrow \mathbf{d}$,

(c) $T^{I Q} \vdash \mathbf{t} \Rightarrow \mathbf{k} \quad T^{I Q} \vdash \mathbf{d} \Rightarrow \mathbf{g}$,

(d) $T^{I Q} \vdash \mathbf{k} \Rightarrow \mathbf{i} \quad T^{I Q} \vdash \mathbf{g} \Rightarrow \mathbf{o}$.

Proof. This proceeds similarly as in [14] using monotonicity of the corresponding evaluative linguistic expressions.

Below, we introduce a 5 -graded cube of opposition with five basic intermediate quantifiers as a generalization of the graded Peterson's square (Fig. 2).

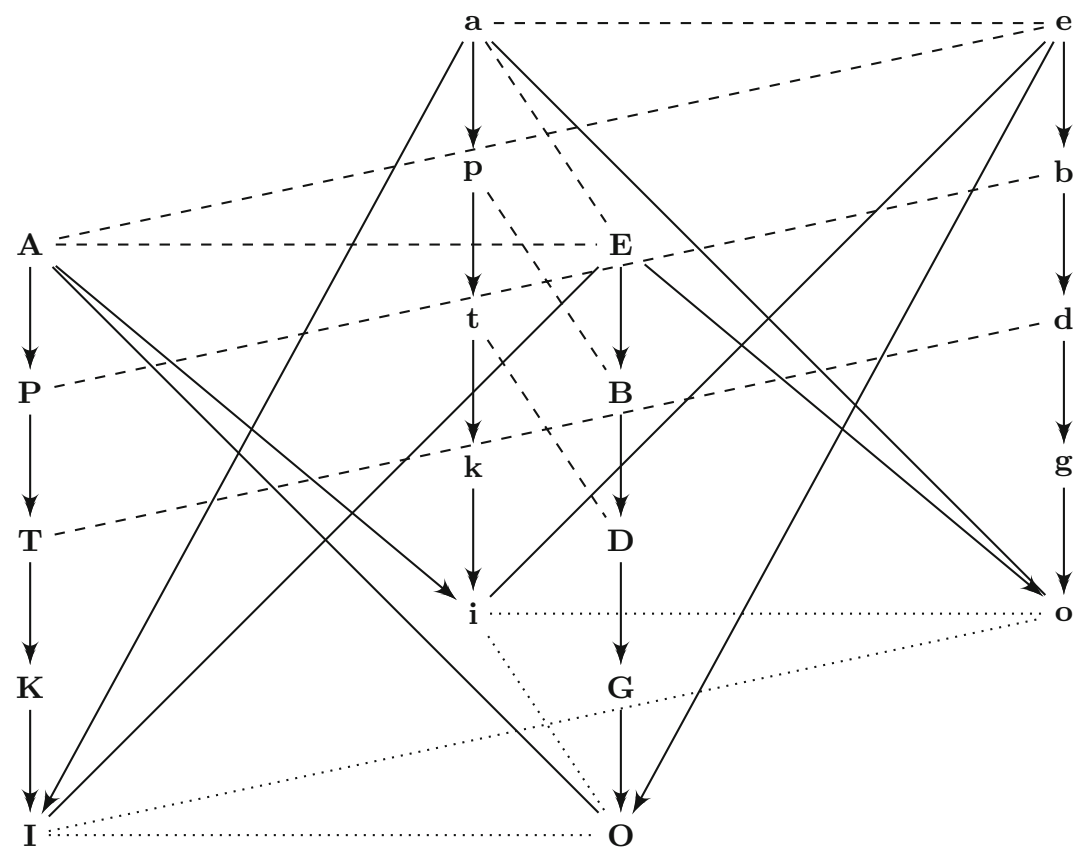

Fig. 2. Graded cube of opposition with generalized intermediate quantifiers

\section{$5 \quad$ Future Applications}

As we mentioned above, the idea of this paper was to introduce new forms of generalized intermediate quantifiers forming graded cube of opposition. An idea for future is to apply the theory of syllogistic reasoning introduced in our previous papers $[6,26]$. Using inferred new forms of valid syllogisms to derive new information which is not included in real data. For example, below we introduce examples of sentences which can be used: 
- Most people who live in an area affected by heavy industry suffer from asthma.

- Almost all shares grow with growing economy.

New idea is to work with examples of natural language linguistic expressions which form graded cube of opposition as follows:

- Most people who do not smoke have higher lung capacity.

- Most children who do not live in an area affected by heavy industry do not suffer from inflammation of the respiratory tract.

\section{Conclusion}

In this paper, we extended the theory of the graded classical Aristotle square of opposition to the graded Aristotle cube of opposition. Furthermore, we suggested a generalization of the Peterson's square of opposition to a graded generalized cube, i.e., the cube whose vertices contain intermediate quantifiers.

The future work will focus on a more detailed analysis of the properties of the graded generalized cube of opposition and to extend by new forms of intermediate quantifiers.

\section{References}

1. Novák, V.: A comprehensive theory of trichotomous evaluative linguistic expressions. Fuzzy Sets and Systems 159(22), 2939-2969 (2008)

2. Novák, V.: Perception-based logical deduction. In: Reusch, B. (ed.) Computational Intelligence. Theory and Applications, pp. 237-250. Springer, Berlin (2005)

3. Novák, V., Lehmke, S.: Logical structure of fuzzy IF-THEN rules. Fuzzy Sets and Systems 157, 2003-2029 (2006)

4. A. Dvořák and M. Holčapek. Type $\langle 1,1\rangle$ fuzzy quantifiers determined by fuzzy measures on residuated lattices. part i. basic definitions and examples. Fuzzy Sets and Systems, 242:31-55, 20014

5. Novák, V.: A formal theory of intermediate quantifiers. Fuzzy Sets and Systems 159(10), 1229-1246 (2008)

6. Murinová, P., Novák, V.: A formal theory of generalized intermediate syllogisms. Fuzzy Sets and Systems 186, 47-80 (2013)

7. Murinová, P., Novák, V.: The structure of generalized intermediate syllogisms. Fuzzy Sets and Systems 247, 18-37 (2014)

8. Thompson, B.E.: Syllogisms using "few","many" and "most". Notre Dame Journal of Formal Logic 23, 75-84 (1982)

9. P.L. Peterson. Intermediate Quantifiers. Logic, linguistics, and Aristotelian semantics. Ashgate, Aldershot, 2000

10. L. Miclet and H. Prade. Analogical proportions and square of oppositions. In: A. Laurent et al. (ed.) Proc. 15th Int. Conf. on Information Processing and Management of Uncer- tainty in Knowledge-Based Systems, July 15-19, Montpellier, CCIS, 443:324-334, 2014

11. Pellissier, R.: "Setting" n-opposition. Logica Universalis 2, 235-263 (2008)

12. Peters, S., Westerståhl, D.: Quantifiers in Language and Logic. Claredon Press, Oxford (2006) 
13. Westerståhl, D.: The traditional square of opposition and generalized quantifiers. Studies in Logic 2, 1-18 (2008)

14. Murinová, P., Novák, V.: Analysis of generalized square of opposition with intermediate quantifiers. Fuzzy Sets and Systems 242, 89-113 (2014)

15. Murinová, P., Novák, V.: The theory of intermediate quantifiers in fuzzy natural logic revisited and the model of "Many". Fuzzy Sets Syst. 388, 56-89 (2020)

16. D. Dubois and H. Prade. From blanche's hexagonal organization of concepts to formal concepts analysis and possibility theory. Logica Universalis, pages 149-169, 2012

17. D. Dubois and H. Prade. Gradual structures of oppositions. In: F. Esteva, L. Magdalena, J.L. Verdegay (eds.) Enric Trillas: Passion for Fuzzy Sets, Studies in Fuzziness and Soft Computing, 322:79-91, 2015

18. Dubois, D., Prade, H., Rico, A.: Graded cubes of opposition and possibility theory with fuzzy events. International Journal of Approximate Reasoning 84, 168-185 (2017)

19. D. Ciucci, D. Dubois, and H. Prade. Oppositions in rough set theory. In: T. Li, H.S. Nguyen, G. Wang, J.W. Grzymala-Busse, R. Janicki, A.E. Hassanien, H. Yu (eds.) Proc. 7th Int. Conf. on Rough Sets and Knowledge Technology (RSKT12), Chengdu, Aug. 17-20, LNCS, 7414:504-513, 2012

20. D. Ciucci, D. Dubois, and H. Prade. The structure of oppositions in rough set theory and formal concept analysis - toward a new bridge between the two settings. In: C. Beierle, C. Meghini (eds.) Proc. 8th Int. Symp. on Foundations of Information and Knowledge Systems (FoIKS14), Bordeaux, Mar. 3-7, LNCS, 8367:154-173, 2014

21. Novák, V.: On fuzzy type theory. Fuzzy Sets and Systems 149, 235-273 (2005)

22. Cignoli, R.L.O., D'Ottaviano, I.M.L., Mundici, D.: Algebraic Foundations of Manyvalued Reasoning. Kluwer, Dordrecht (2000)

23. Novák, V., Perfilieva, I., Močkoř, J.: Mathematical Principles of Fuzzy Logic. Kluwer, Boston (1999)

24. Andrews, P.: An Introduction to Mathematical Logic and Type Theory: To Truth Through Proof. Kluwer, Dordrecht (2002)

25. Novák, V., Perfilieva, I., Dvořák, A.: Insight into Fuzzy Modeling. Wiley \& Sons, Hoboken, New Jersey (2016)

26. P. Murinová. Generalized intermediate syllogisms with more premises. In Proc. FUZZ-IEEE 2019, New Orleands, USA, 2019 